\title{
MASS LOSS AND EVOLUTION OF MASSIVE STARS IN THE MAGELLANIC CLOUDS
}

\author{
CLAUS LEITHERER ${ }^{1}$, NORBERT LANGER ${ }^{2}$, \\ 1 STScI, 3700 San Martin Drive, Baltimore, MD 21218. Affiliated with the Astrophysics \\ Division, Space Science Department of ESA. \\ 2 Universitätssternwarte, Geismarlandstrasse 11, D-3400 Göttingen, Germany
}

\section{Introduction}

The structure and evolution of massive stars is significantly influenced by effects of chemical composition in a low-metallicity environment (as compared to the solar neighbourhood, SN), such as the Magellanic Clouds. A fundamental ingredient in evolutionary models is the stellar mass-loss rate $\dot{M}$. Lower metal content decreases the mass-loss rates derived theoretically, which in turn affects the stellar evolution models. On the other hand, different evolutionary models predict different stellar parameters (especially $L$ ), which again influence $\dot{M}$ so that an iterative procedure is required to achieve self-consistency.

Here we report on the first results obtained from such iterative models and address two issues: mass loss as a function of chemical composition on the main sequence and the mechanism for the radius- and $\dot{M}$-variability in Luminous Blue Variables (LBVs).

\section{The influence of the chemical composition on MS evolution}

We computed a set of ZAMS models with chemical composition appropriate for the SN and the Magellanic Clouds. The helium abundance $Y$ is related to the metal abundance $Z$ by $Y(Z)=$ $Y_{\text {primordial }}+\frac{d Y}{d Z} Z$. Observations and models for the chemical evolution of galaxies suggest $Y_{\text {primordial }}=0.24$ and $\frac{d Y}{d Z}=2$. The resulting ZAMSs are shown in Fig 1. Taking $Z_{S M C}=0.1 Z_{\theta}$, we find the ZAMS of the SMC to be hotter than that of the solar neighbourhood by $\sim 10 \%$. Accordingly, O stars close to the ZAMS in the SMC should be classified earlier, by about one subtype, than their counterparts in the SN with the same mass. This effect is relevant when the initial mass function for such stars is derived. We note that the results shown in Fig 1 are largely independent of $\frac{d Y}{d Z}$ as long as $\frac{d Y}{d Z} \leqslant 2$.

Wind models with parameters resulting from our stellar models have been computed for the ZAMS. As expected, a lower metal content leads to lower theoretical mass-loss rates. Fig 2 illustrates the functional dependence of $\dot{M}$ on $Z$. We find $\dot{M} \sim Z^{0.6-0.7}$. Fig 2 also includes the results we get if $\frac{d Y}{d Z}=1$ is used in our stellar models. For $Z>Z_{0}, Y$ is notably lower (as is $L$ ) so that $\dot{M}$ is lower.

Kudritzki et al. (1987) found $\dot{M} \sim Z^{0.47}$ from models for one $05 \mathrm{~V}$ star with $0.1 Z_{\odot} \leq Z_{\leq} \mathrm{Z}_{\Theta}$. Our more extensive grid of models implies a somewhat larger exponent. Furthermore, the functional behaviour is more complex than a simple power law. 
We studied the behaviour of the theoretical $\dot{M}$ versus $L, T_{\text {eff }}$ relation along the MS using Maeder \& Meynet's (1988) evolutionary tracks. Applying a scaling factor for $\dot{\bar{M}}_{M S} / \dot{M}_{Z A M S}$ to our ZAMS models we derive the total mass lost on the MS as a function of $Z$. Assuming MS evolution is unaffected by mass loss, if less than $5 \%$ of the initial mass is lost, a relation is found between the ZAMS mass below which mass loss is unimportant on the MS $\left(M_{c r i t}\right)$ and $Z$. This is shown in the insert to Fig 1). Taking $Z_{S M C}=0.1 Z_{\text {e }}$, we conclude that mass loss on the MS is unimportant for SMC stars below $\mathrm{M} \approx 80 M_{\mathrm{e}}$.

\section{The radius- and mass-loss variability of LBVs}

Our models predict mass loss to be relatively unimportant on the MS. But mass loss is a determining factor for stars in the post-MS, above all in the LBV phase. R71 and R110 are two LBVs of the LMC discussed by Leitherer et al. (1989) and Stahl et al. (1990), both having $L \approx 10^{5.5} L_{\theta}$ $\approx$ constant. R.71 has been found to vary in $T_{\text {eff }}$ from $\sim 18,000 \mathrm{~K}$ to $\sim 9,000 \mathrm{~K}$ with $M$ increasing by 1-2 orders of magnitude. In contrast, $T_{\text {eff }}$ of R110 has decreased from $10,000 \mathrm{~K}$ to 7,500 K but with $\dot{M}$ decreasing by a large factor. We computed a set of wind models and can explain the wind properties of both stars by radiation pressure. If $18,000 \mathrm{~K} \lessgtr T_{\text {eff }} \leq 8,000 \mathrm{~K}$, a decrease in temperature leads to a recombination from weak Fe III to strong Fe II lines, and a large increase in $\dot{M}$ results. On the other hand, if $T_{\text {eff }} \leqslant 8,000 \mathrm{~K}$, a decrease in $T_{\text {eff }}$ produces lower $\dot{M}$ because of decreasing photospheric flux in the Balmer continuum despite the presence of strong lines.

These results lead us to conclude that the observed variations of $T_{\text {eff }}$ (and thus of the radius) are not a consequence of a variation of the wind density as has been suggested by Davidson (1987). We propose that R71 and R110 are post-RSGs evolving to the blue part of the HRD on a thermal time-scale. Around $T_{\text {eff }} \approx 8,000 \mathrm{~K}$ these stars meet a phase of very strong mass loss induced by radiation pressure $\left(\dot{M} \approx 10^{-4} M_{\Theta} \mathrm{yr}^{-1}\right)$ which can be kept until the ionization conditions in the wind change at $\sim 18,000 K$. Consequently, the star rapidly evolves toward blue and then toward red, due to thermal relaxation. The envelope mass of a star with $M_{Z A M S} \approx 25-30 M_{\odot}$ is $\sim 2 M_{\Theta}$ leading to a relaxation time-scale of $\sim 10 y r$, well in agreement with LBV observations.

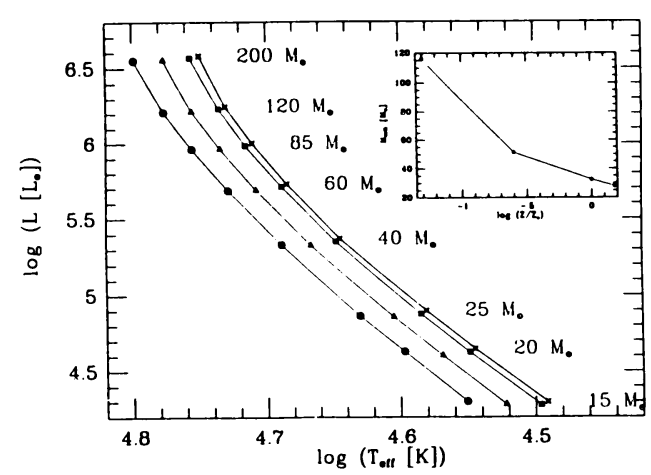

Figure 1. Theoretical ZAMS for $Z=$ $1.5 Z_{\theta}, Z_{\theta}, 0.25 Z_{\theta}, 0.05 Z_{\theta}$. Lower $Z$ results in higher $T_{\text {eff }}$. Insert: initial mass below which MS- $\dot{M}$ is unimportant, vs. $Z$.

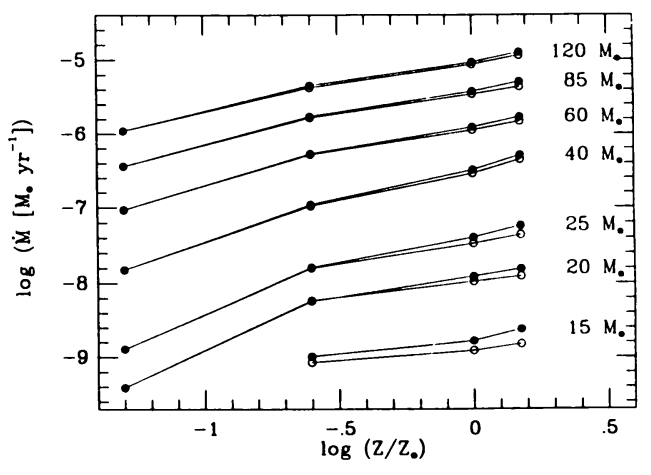

Figure $2 . \dot{M}$ vs. $Z$ on the ZAMS. Full circles: $\frac{d Y}{d Z}=2$; open circles: $\frac{d Y}{d Z}=1$. 


\section{References}

Davidson, K. (1987), Astrophys J. 317, 760.

Kudritzki, R.P., Pauldrach, A., Puls, J. (1987), Astron. Astrophys. 173, 293.

Leitherer, C., Schmutz, W., Abbott, D.C., Hamann, W-R., Wessolowski, V. (1989), Astrophys. J. 346, 919.

Maeder, A., Meynet, G. (1988), Astron. Astrophys. Suppl. Ser. 76, 411.

Stahl, O., Wolf, B., Klare, G., Jüttner, A., Cassatella, A. (1990), Astron. Astrophys. 228, 379. 